\title{
Linear Spaces on the Intersection of Cubic Hypersurfaces
}

\author{
By
}

\section{J. Lewis*, Michigan, and Rainer Schulze-Pillot**, Göttingen}

(Received 16 August 1983; in revised form 2 April 1984)

Abstract. Upper bounds for the number of variables necessary to imply the existence of an $m$-dimensional linear variety on the intersection of $r$ cubic hypersurfaces over local and global fields are given.

R. BRAUER [5] demonstrated that if a field $F$ has the property that there exists an integer valued function $\gamma_{F}(d)$ such that every additive equation $\sum_{1}^{\gamma_{F}(d)} a_{i} x_{i}^{d}=0, a_{i} \in F$, has a nontrivial zero defined over $F$, then there exists a function $\Phi_{F}\left(d_{1}, \ldots, d_{r} ; m\right)$ such that every projective variety $V \subset \mathbb{P}^{n}$ which is the common zero set of $r$ forms in $n+1$ variables of degrees $d_{1}, \ldots, d_{r}$ with coefficients in $F$ necessarily contains a projective $m$-dimensional linear $F$ space provided $n \geqslant \Phi_{F}\left(d_{1}, \ldots, d_{r} ; m\right)$, and the conclusion fails for smaller $n$. Since it is easily demonstrated that $\gamma_{\mathbb{Q}_{p}}(d)=\gamma_{p}(d)$ exists for the $p$-adic field $\mathbb{Q}_{p}$, and indeed $\gamma_{p}(d) \leqslant d^{2}+1$ for all $p$ and $d$ (see [7]), it follows that $\Phi_{p}\left(d_{1}, \ldots, d_{r} ; m\right)=\Phi_{\mathbb{Q}_{p}}\left(d_{1}, \ldots, d_{r} ; m\right)$ exists. The argument as presented by R. Brauer did not provide estimates for $\Phi_{p}\left(d_{1}, \ldots, d_{r} ; m\right)$ and simple minded calculations suggested by his argument would provide upper bounds on $\Phi_{p}$ of excessively high exponential order. Over the last 40 years considerable effort has been expended on obtaining bounds for $\Phi_{p}$ when $m=0$. We mention only a few of the results that have been obtained. Let $\Phi_{p}\left(d_{1}, \ldots, d_{r}\right)=\Phi_{p}\left(d_{1}, \ldots, d_{r} ; 0\right)$. Then $\Phi_{p}(2)=4$ (MEYER [16]); $\Phi_{p}(3)=9$ (LEWIS [14]); $\Phi_{p}(2,2)=8$ (DEMYANOV [9], Birch, LeWIS and MurPhy [3]); $\Phi_{p}(4) \geqslant 20$ (TERJANIAN $[21]) ; \Phi_{p}\left(d_{1}, \ldots, d_{r}\right)=d_{1}^{2}+\ldots+d_{r}^{2}$ provided $p$ is sufficiently large

*D. J. Lewis was partially supported by a National Science Foundation grant.

** Rainer Schulze-Pillot was supported by a Deutsche Forschungsgemeinschaft Stipendium. 
compared to the $d_{i}$ (Ax and KocheN [2]); for each prime $p$ and each $\varepsilon>0$ there exist infinitely many $d$ for which $\Phi_{p}(d)>e^{d^{1-\varepsilon}}$ (ARCHIPOV and Karacuba [1], Lewis and MonTgOMERY [15], BRownawell [6]), $\Phi_{p}(d) \ll e^{d^{2}}$ (SCHMIDT [17]). In the last year David Leep and Wolfgang Schmidt have shown $\Phi_{p}(2, \ldots, 2) \leqslant 2 r^{2}+2 r-4$ (LEEP [12]); $\Phi_{p}(3, \ldots, 3) \leqslant 50,000 r^{3}$ (SCHMIDT [18]). Very little investigation has occured for the situation where $m>0$.

B. J. BIRCH [4] refined Brauer's argument and obtained an analogous result for varieties defined by forms of odd degree over $\mathbb{Q}$. Specifically, Birch showed one can define a function $\Gamma_{\mathbb{Q}}\left(d_{1}, \ldots, d_{r} ; m\right)=$ $=\Gamma\left(d_{1}, \ldots, d_{r} ; m\right)$ with all arguments $d_{i}$ odd with the properties ascribed to $\Phi$. Little is known regarding the size of $\Gamma$ and what is known concerns cubic hypersurfaces; specifically: $9 \leqslant \Gamma(3 ; 0) \leqslant 15$ (DAVENPORT $[8]) ; \Gamma(3 ; 0)=9$ if the hypersurface is nonsingular (Heath-Brown [10]); and $\Gamma(3, \ldots, 3 ; 0) \leqslant(10 r)^{5}$ (Schmidt [19]). For odd $d_{i}$ ScHMidT [20] has shown a basis $\mathbf{x}^{(0)}, \ldots, \mathbf{x}^{(m)}$ can be chosen for the linear space which have integer coordinates of small absolute value, say $\leqslant N$, provided $n$ exceeds a function of $d_{1}, \ldots, d_{r}, N$ and the coefficients of the forms. The function provided by the proof would be astronomical in size.

It is our purpose here to draw attention to the case $m>0$ and to give bounds for $\Phi_{p}(3, \ldots, 3 ; m)=\lambda_{p}(r, m)$ and for $\Gamma(3, \ldots, 3 ; m)=$ $=\Lambda(r, m)$, when $m>0$. Except for one use of a result of Schmidt our methods are elementary and on the whole our results are disappointingly weak.

\section{Proposition 1.}

$$
\Phi_{p}(2, \ldots, 2 ; m) \leqslant \begin{cases}4+2 m & \text { if } r=1, \\ 2 r^{2}+2 r-4+(r+1) m & \text { if } r>1 .\end{cases}
$$

Proof. The proof is by induction on $m$ for fixed $r$. When $r \geqslant 1$ and $m=0$ the conclusion is Theorem 2.8 of LEEP [12]. For $m>0$, the conclusion follows from Corollary 2.4 of [12].

Proposition 2.

$$
\Phi_{p}(3 ; m) \leqslant \begin{cases}9 & \text { if } m=0, \\ 3 m^{2}+11 m+5 & \text { if } m \geqslant 1 .\end{cases}
$$

Proof. Again the proof is by induction on $m$. If $m=0$, the conclusion holds (LEwIS [14]). Let $\psi(m)$ denote the function on the right hand side of the inequality. Suppose the proposition holds for 
some integer $m$. Let $f$ be a cubic form in $n+1$ variables over $\mathbb{Q}_{p}$ with $n \geqslant \psi(m+1)$. Since $\psi$ is monotonic it follows that the zero set $V$ of $f$ contains an $m$-dimensional $\mathbb{Q}_{p}$-linear projective space $\mathscr{M}$. After a change of variable we may assume

Then

$$
\mathscr{M}=\left\{t_{0} \mathbf{e}_{0}+\ldots+t_{m} \mathbf{e}_{m} \mid \mathbf{t} \in \mathbb{P}_{\mathbb{Q}_{p}}^{m}\right\}
$$

$$
\begin{gathered}
f\left(t_{0} \mathbf{e}_{0}+\ldots+t_{m} \mathbf{e}_{m}+t\left(0 \ldots 0, y_{m+1}, \ldots, y_{n}\right)\right)= \\
\quad=\sum_{i, j=0}^{m} t_{i} t_{j} t L_{i j}(\mathbf{y})+\sum_{i=0}^{m} t_{i} t^{2} Q_{i}(\mathbf{y})+t^{3} C(\mathbf{y}),
\end{gathered}
$$

where the $L_{i j}, Q_{i}, C$ are respectively linear, quadratic and cubic forms. By Proposition 1 there is a 9-dimensional $\mathbb{Q}_{p}$-projective linear space $\mathscr{N}$ on $L_{00}=\ldots=L_{m m}=Q_{0}=\ldots=Q_{m}=0$ provided

$$
n-(m+1)^{2} \geqslant 2(m+1)^{2}+2(m+1)-4+9(m+2) ;
$$

i. e., provided

$$
n \geqslant 3(m+1)^{2}+11(m+1)+5=\psi(m+1) .
$$

It follows that $C$ vanishes on a point $\mathbf{a}$ in $\mathscr{N}$ and hence $\left\{t_{0} \mathbf{e}_{0}+\right.$ $\left.+\ldots+t_{m} \mathbf{e}_{m}+t \mathbf{a}\right\}$ is a $(m+1)$-dimensional $\mathbb{Q}_{p}$-linear projective space on $f=0$.

Theorem 1. There exist constants $C_{0}, C_{1}, C_{2}$ such that

$$
\lambda_{p}(r, m) \leqslant \begin{cases}\mathrm{C}_{0} r^{3} & \text { if } m=0, \\ C_{1} r^{2} m^{2}+C_{2} r^{4} m & \text { if } m \geqslant 1 .\end{cases}
$$

Proof. The assertion for $m=0$ is a theorem of ScHMIDT [18]. For $m \geqslant 1$, the assertion follows by induction on $m$, as above, except this time we need find a projective $\mathbb{Q}_{p}$-linear space of dimension $\geqslant C_{0} r^{3}$ on the intersection of $(m+1)^{2} r$ linear forms and $(m+1) r$ quadratic forms.

We next prove a theorem that provides upper bound estimates for $\Gamma(3, m)=\Phi_{\mathbb{Q}}(3 ; m)$. Actually we prove a result for any field $F$ with the property that $\Phi_{F}(3 ; 0)$ exists. One cannot argue as in Proposition 2 since $\Phi_{F}(3 ; 0)$ may be defined while $\Phi_{F}(2 ; 0)$ may not; as is the case when $F=\mathbb{Q}$. The argument we use is based on an idea of BIRCH [4].

Theorem 2. Let $F$ be a field with the property that there is a constant $\lambda_{0}=\lambda_{0}(F)$ such that every cubic projective hypersurface defined over $F$ 
and lying in $\mathbb{P}^{n}$ contains a point defined over $F$ provided $n \geqslant \lambda_{0}$. Then with $\alpha=\frac{1}{2}(5+\sqrt{17})$, we have

$$
\Phi_{F}(3 ; m) \leqslant A(m+1)^{\alpha},
$$

where $A$ is a constant that depends only on $\lambda_{0}$.

Note: In the case of the rational field, DAVENPORT [8] has shown $\lambda_{0}(\mathbb{Q}) \leqslant 15$. Hence Theorem 2 provides an upper bound for $\Gamma(3 ; m)$.

Proof. It will be convenient to work affinely rather than projectively. Let $C(\mathbf{x})$ be a cubic form in $N$ variables defined over $F$. Let $T(\mathbf{x}, \mathbf{y}, \mathbf{z})$ be a trilinear form on $F^{N}$ with $T(\mathbf{x}, \mathbf{x}, \mathbf{x})=C(\mathbf{x})$. Here $T$ need not be symmetric and indeed is not if the characteristic of $F$ is 2 or 3. Let $\delta(u+1)=\Phi_{F}(3 ; u)$ and suppose $\delta(u)<\infty$ for $1 \leqslant u<m$. Clearly $\delta(u)$ is monotonic increasing. Let $t$ be a fixed positive integer less than $m$. Let

$$
\begin{gathered}
s=\delta(t)+3(m-t)^{2}+1, \\
\Delta=\delta(m-t)+3 s^{2}+s .
\end{gathered}
$$

Suppose that $N>\Delta$. Let $W$ be an arbitrary, but fixed, $F$-linear subspace of $F^{N}$ of dimension $s$. Let $\mathbf{a}_{1}, \ldots, \mathbf{a}_{s}$ be a basis for $W$. Since $N>\Delta$, the $3 s^{2}$ linear equations

$$
T\left(\mathbf{a}_{i}, \mathbf{a}_{j}, \mathbf{x}\right)=T\left(\mathbf{a}_{i}, \mathbf{x}, \mathbf{a}_{j}\right)=T\left(\mathbf{x}, \mathbf{a}_{i}, \mathbf{a}_{j}\right)=0, \quad 1 \leqslant i, j \leqslant s,
$$

have a $F$-linear space of solutions $U$ such that $U \cap W=\{0\}$ and $\operatorname{dim} U>\delta(m-t)$. By the definition of $\delta(m-t)$ the form $C$ vanishes identically on a $F$-linear subspace $U_{1}$ of $U$ with $\operatorname{dim} U_{1}=m-t$. Let $\mathbf{b}_{1}, \ldots, \mathbf{b}_{m-t}$ be a basis for $U_{1}$. By the definition of $s$, the $3(m-t)^{2}$ linear equations

$$
T\left(\mathbf{b}_{i}, \mathbf{b}_{j}, \mathbf{x}\right)=T\left(\mathbf{b}_{i}, \mathbf{x}, \mathbf{b}_{j}\right)=T\left(\mathbf{x}, \mathbf{b}_{i}, \mathbf{b}_{j}\right)=0, \quad 1 \leqslant i, j \leqslant m-t,
$$

have a $F$-linear subspace of solutions $W_{1}$ lying in $W$ with $\operatorname{dim} W_{1}>\delta(t)$. By the definition of $\delta(t)$, the form $C$ vanishes on a $F-$ linear subspace $W_{2}$ of $W_{1}$ with $\operatorname{dim} W_{2}=t$. Since $W_{2} \cap U_{1} \subset W \cap U=\{\boldsymbol{0}\}$ we have $V^{\prime}=W_{2}+U_{1}$ is of dimension $m$. Our construction is such that $C$ vanishes identically on $V^{\prime}$.

Thus we have shown, if for some $t$ in $1 \leqslant t<m$,

$$
N \geqslant \delta(m-t)+3\left\{\delta(t)+3(m-t)^{2}+1\right\}^{2}+\delta(t)+3(m-t)^{2}+2,
$$

then $C=0$ contains a $F$-linear space of dimension $m$. It follows that 


$$
\delta(m) \leqslant \delta(m-t)+3\left\{\delta(t)+3(m-t)^{2}+2\right\}^{2}, \quad 1 \leqslant t<m .
$$

Let $m=q t+u$ where $0 \leqslant u<t$. Then we have

$$
\begin{gathered}
\delta(m) \leqslant \delta(m-2 t)+3\left\{\delta(t)+3(m-t)^{2}+2\right\}^{2}+ \\
+3\left\{\delta(t)+3(m-2 t)^{2}+2\right\}^{2}
\end{gathered}
$$

and, by induction, since $\delta(u) \leqslant \delta(t)$ we obtain

whence

$$
\delta(m) \leqslant \delta(t)+3 \sum_{j=1}^{[m / t]}\left\{\delta(t)+3 j^{2} t^{2}+2\right\}^{2}, \quad 1 \leqslant t<m
$$

$$
\delta(m) \leqslant 3 \frac{m}{t} \delta(t)^{2}+\frac{G(m, t)}{t} \delta(t)+\frac{U(m, t)}{t}, \quad 1 \leqslant t<m,
$$

where $G$ and $U$ are cubic and quintic forms, respectively, with coefficients independent of $m, t$. Thus, there exists an absolute constant $C \geqslant 1$ such that,

$$
\delta(m) \leqslant C \frac{m}{t}\left\{\delta(t)^{2}+m^{2} \delta(t)+m^{4}\right\}, \quad 1 \leqslant t<m .
$$

With $\eta(m)=\frac{\delta(m)}{m^{\alpha}}$ for $m>1$ we obtain

$$
\eta(m)<2 C \frac{m^{1-\alpha}}{t}\left(\delta(t)^{2}+m^{4}\right)
$$

for $1 \leqslant t<m$. Taking $t=1$ we get

$$
\eta(m) \ll m^{5-\alpha} \text {. }
$$

Substituting $\eta(t) \ll t^{5-\alpha}$ into (2) we find that

$$
\eta(m) \ll m^{1-\alpha} t^{9}+m^{5-\alpha} t^{-1} .
$$

With $t=\left[m^{2 / 5}\right]$ this becomes

$$
\eta(m) \ll m^{(23 / 5)-\alpha}=m^{(2 / \alpha)-(2 / 5)} .
$$

We may suppose that $C \geqslant 1$ in (2). Put

$$
\begin{gathered}
\eta^{*}(m)=\max \left(100 C^{2}, \eta(1), \ldots, \eta(m-1)\right), \\
t=t(m)=\left[8 \mathrm{Cm}^{2 / \alpha} / \eta^{*}(m)\right] .
\end{gathered}
$$

When $m$ is large, we have $2 \leqslant t<m$ by (3). We insert $t=t(m)$ into (2) to obtain 


$$
\begin{gathered}
\eta(m) \leqslant 2 C\left(\eta^{*}(m)^{2} m^{1-\alpha}\left(8 C m^{2 / \alpha} / \eta^{*}(m)\right)^{2 \alpha-1}+\right. \\
\left.+2 m^{5-\alpha-(2 / \alpha)} \eta^{*}(m) / 8 C\right)<\eta^{*}(m)\left((8 C)^{2 \alpha} \eta^{*}(m)^{2-2 \alpha}+\frac{1}{2}\right) .
\end{gathered}
$$

But $\eta^{*}(m) \geqslant(10 C)^{2}$, so that $\eta^{*}(m)^{2 \alpha-2}>\eta^{*}(m)^{6}>(10 C)^{10}>$ $>(10 C)^{2 \alpha}>2(8 C)^{2 \alpha}$.

\section{Therefore}

$$
\eta(m)<\eta^{*}(m)
$$

and $\eta^{*}(m+1)=\eta^{*}(m)$. Thus for large $m$ the function $\eta^{*}(m)$ is constant, and $\eta(m)$ is bounded. Thus $\delta(m) \ll m^{\alpha}$ for $m \geqslant 1$, and $\delta(m) \leqslant A(m+1)^{\alpha}$ for $m \geqslant 0$.

Our original version of Theorem 2 contained an additional factor $(\log m)^{B}$. The improved version given here is due to the referee.

To compute an upper bound for $\Lambda_{F}(r, m)=\Phi_{F}(3, \ldots, 3 ; m)$, where $\lambda_{0}=\Phi_{F}(3 ; 0)$ exists, one can proceed as in the proof of Theorem 2 . If $A_{F}(r, m)$ is the number of variables needed for $r$ cubic forms over $F$ to contain a common $m$-dimensional $F$-linear space of zeros, we find that for any $t$ in $1 \leqslant t<m$,

$$
\Delta_{F}(r, m) \leqslant \Delta_{F}(r, t)+3 r \sum_{1}^{[M / t]}\left\{\Delta_{F}(r, t)+3 r j^{2} t^{2}+2\right\}^{2}
$$

whence, for $1 \leqslant t<m$, we have

$$
\Delta_{F}(r, m) \leqslant C \frac{r m}{t}\left\{\Delta_{F}(r, t)^{2}+r m^{2} \Delta_{F}(r, t)+r^{2} m^{4}\right\} .
$$

To proceed further one must know something regarding the size of $\Delta_{F}(r, 1)$.

In case the field is the rational field, we know from [19] that $\Delta_{\mathbb{Q}}(r ; 1) \leqslant a r^{5}$ for some absolute constant $a$. In that case with $t=1$, we obtain

$$
\Delta_{\mathbb{Q}}(r ; m) \leqslant A r^{11} m+B r^{3} m^{5}
$$

where $A, B$ are absolute constants. We have been unable to improve on this by elementary methods.

Wolfgang Schmidt outlined for us orally the following result, which we include with his permission.

Theorem 3 (W. Schmidt). $A_{\mathbb{R}}(r, m) \ll r m^{2}$.

Schmidt's argument is as follows. Let $C_{1}(\mathbf{x}), \ldots, C_{r}(\mathbf{x})$ be $r$ cubic forms over $\mathbb{R}$ in $r+1$ variables. Then the map 


$$
f(\mathbf{x})=\left(C_{1}(\mathbf{x}), \ldots, C_{r}(\mathbf{x})\right)
$$

is a continuous map of the $r$-dimensional sphere into $\mathbb{R}^{r}$. It follows from the Borsuk-Ulam Theorem ${ }^{1}$ that there exists a point a on the sphere (hence $\mathbf{a} \neq \mathbf{0}$ ) such that $f(\mathbf{a})=f(-\mathbf{a})$. Since $f$ is an odd degree map, we have $f(\mathbf{a})=0$. Thus $\Lambda_{\mathbb{R}}(r, 0)=r$.

The real manifold $M$ of common zeros of $r$ cubic forms in $n$ variables meets every $r+1$ dimensional real manifold and hence $\operatorname{dim} M \geqslant n-r$.

Let $C_{1}, \ldots, C_{r}$ be $r$ cubic forms in $n+1$ variables with real coefficients. Let $T_{v}(\mathbf{x}, \mathbf{y}, \mathbf{z})$ be the symmetric trilinear forms on $\mathbb{R}^{n+1}$ such that $T_{v}(\mathbf{x}, \mathbf{x}, \mathbf{x})=C_{v}(\mathbf{x})$. Let $\mathbf{x}_{1}, \ldots, \mathbf{x}_{2 m}$ be $(n+1)$-tuples with distinct variables and form the cubic forms $T_{v}\left(\mathbf{x}_{i}, \mathbf{x}_{j}, \mathbf{x}_{k}\right)=T_{v i j k}$, with $1 \leqslant v \leqslant r, 1 \leqslant i, j, k \leqslant 2 m$. There are $r\left(\begin{array}{c}2 m+2 \\ 3\end{array}\right)$ of the forms $T_{\nu i j k}$ and hence the real manifold $\mathscr{T}$ of common zeros of the $T_{v i j k}$ in $\mathbb{R}^{2 m(n+1)}$ is of dimension $\geqslant 2 m(n+1)-r\left(\begin{array}{c}2 m+2 \\ 3\end{array}\right)$. A vector $\mathbf{y}$ in $\mathbb{R}^{2 m(n+1)}$ determine $2 m$ vectors in $\mathbb{R}^{n+1}$ by the association $\mathbf{y}=\left(\mathbf{y}_{1}, \ldots, \mathbf{y}_{2 m}\right)$. The set $\mathscr{Y}$ of vectors $\mathbf{y}$ in $\mathbb{R}^{2 m(n+1)}$ such that the rank of $\left\{\mathbf{y}_{1}, \ldots, \mathbf{y}_{2 m}\right\}$ is at most $m$ is a real manifold of dimension $\Delta=m(n+1)+m^{2}$; indeed $\mathscr{Y}$ is the union of linear $\mathbb{R}$ manifolds of dimension at most $\Delta$. Hence, if $2 m(n+1)-r\left(\begin{array}{c}2 m+2 \\ 3\end{array}\right)>m(n+1)+m^{2}$ there are points $\mathbf{a}=$ $=\left(\mathbf{a}_{1}, \ldots, \mathbf{a}_{m}\right)$ on $\mathscr{T}$ not on $\mathscr{Y}$. Thus if $n>m+\frac{2}{3} r(m+1)(2 m+1)$ there exist $m+1$ linear independent points $\mathbf{b}_{0}, \ldots, \mathbf{b}_{m}$ in $\mathbb{R}^{n+1}$ such that $T_{v}\left(\mathbf{b}_{i}, \mathbf{b}_{j}, \mathbf{b}_{k}\right)=0,1<v<r, 0<i, j, k<m$; whence $C\left(t_{0} \mathbf{b}_{0}+\ldots+\right.$ $\left.+t_{m} \mathbf{b}_{m}\right)$ is identically zero. Thus $\Lambda_{\mathbb{R}}(r, m)<2 r m^{2}$ if $m \geqslant 5$ and $A_{\mathbb{R}}(r, m)<5 r m^{2}$ if $m \geqslant 1$.

An adaptation of this method for the field $\mathbb{Q}$ would yield $\Lambda_{Q}(r, m) \ll r^{5} m^{14}$ which is better than the bound obtained above if $r$ is very large compared to $m$.

Since we have shown $\Lambda_{\mathbb{Q}_{p}}(r, m) \ll r^{4} m^{2}$ and $A_{\mathbb{R}}(r, m) \ll r m^{2}$, it is disappointing to have to settle for $A_{\mathbb{Q}}(r, m) \leqslant A r^{11} m+B r^{3} m^{5}$ resp. $\Lambda_{\mathbb{Q}}(r, m) \ll r^{5} m^{14}$.

1 An algebraic proof of this theorem for polynomial mappings of odd degree has been given by KNEBUSCH [11]. 
If one were to make full use of the local information it would appear some recourse to the Hardy-Littlewood method or adelic methods will be needed. To date we have been unsuccessful in adopting such methods to determining linear manifolds on algebraic varieties.

It is easily seen that the proof of Theorem 3 can be adapted to give

Theorem 4 (W. Schmidt). Let $\Lambda_{R}(r, d ; m)$ be the least number such that the zero set of any $r$ forms of odd degree $d$ with real coefficients in $s>\Lambda_{\mathbb{R}}(r, d ; m)$ variables contains an $m$-dimensional linear projective real space. Then $\Lambda_{\mathbb{R}}(r, d ; m)<C(d) r m^{d-1}$, where $C(d)$ is a constant that depends on $d$.

\section{References}

[1] ARCHIPOV, G. I., KARACUBA, A. A.: On local zeros of forms (In Russian). Izv. Akad. Nauk. Ser. Mat. 45, No. 5, 948-961 (1981).

[2] AX, J., KoCHEN, S. : Diophantine problems over local fields I. Amer. J. Math. 87, 605-630 (1965).

[3] Birch, B. J., Lewis, D. J., Murphy, T. G.: Simultaneous quadratic forms. Amer. J. Math. 84, 110-115 (1962).

[4] BIRCH, B. J.: Homogeneous forms of odd degree in a large number of variables. Mathematika 4, 102-105 (1957).

[5] BRAUER, R.: A note on systems of homogeneous algebraic equations. Bull. Amer. Math. Soc. 51, 749-755 (1945). (1984).

[6] Brownawell, D.: On p-adic zeros of forms. J. Number Th. 18, 342-349

[7] Davenport, H., Lewis, D. J.: Homogeneous additive equations. Proc. Royal Soc. London 274 A, 443-460 (1963).

[8] Davenport, H.: Cubic forms in 16 variables. Proc. Royal Soc. London 272 A, 285-303 (1963).

[9] Dem'Yanov, V.B.: Pairs of quadratic forms over complete fields (In Russian. Izv. Akad. Nauk SSSR Ser. Mat. 20, 307-324 (1956).

[10] Heath-Brown, R.: Cubic forms in 10 variables. Proc. London Math. Soc. (3) 47, 225-257 (1983).

[11] KNEBUSCH, M.: An algebraic proof of the Borsuk-Ulam theorem for polynomial mappings. Proc. Amer. Math. Soc. 84, 29 -32 (1982).

[12] LEEP, D.: Systems of quadratic forms. To appear.

[13] LEEP, D., SCHMIDT, W.: Systems of homogeneous equations. Invent. Math. 71, $539-549$ (1983).

[14] LEWIS, D. J. : Cubic homogeneous equations over $p$-adic number fields. Ann. Math. 56, 473-478 (1952).

[15] Lewis, D. J., Montgomery, H. L.: On zeros of $p$-adic forms. Michigan Math. J. 30, 83-87 (1983).

[16] MEYER, A.: Vierteljahrsschrift Naturforsch. Gesellsch. Zürich 29, 209-222

[17] SchmidT, W.: The solubility of certain p-adic equations. J. Number Th. 19, $63-80$ (1984).

[18] SCHMIDT, W.: On cubic polynomials: III. Systems of $p$-adic equations. Mh. Math. 93, 211-223 (1982). 
[19] SchmidT, W.: On cubic polynomials: IV. Systems of rational equations. Mh. Math. 93, 329-348 (1982).

[20] SCHMIDT, W.: Diophantine inequalities for forms of odd degree. Adv. Math. 38, 128-151 (1980).

[21] TERJANIAN, G.: Formes $p$-adiques anisotropes. J. Reine Angew. Math. 313, 217-220 (1980).

R. SCHULZE-PILLOT

Freie Universität Berlin

Institut für Mathematik II

Arnimallee 3

D-1000 Berlin (West) 33

and

D. J. LEWIS

Department of Mathematics

University of Michigan

Ann Arbor, MI 48109, U.S.A. 